\title{
一些特异生境微生物的化学成分及其抗肿瘤活性研究
}

\author{
李瑶瑶沈月毛* \\ (山东大学药学院 济南 250012)
}

\begin{abstract}
摘要 癌症发病率和耐药性的不断攀升, 促使新结构和新作用机制药物的发现更为迫切. 近年来, 特异生境微生物的 化学成分和生物活性研究日益受到关注. 本课题组通过对药用植物内生菌和海洋微生物的分离、篮选和系统的化学成 分分离, 获得了一些结构新颖复杂的活性天然产物, 并对其中 2 个化合物的抗肿瘤作用机制进行了研究. 这些结果表 明特异生境微生物蕴含着丰富的新活性化学成分, 值得深入发掘.
\end{abstract}

关键词 植物内生菌; 海洋微生物; 天然产物; 抗肿瘤作用

\section{Chemical Components of Some Microorganisms Isolated from Specific Habitats and Their Antitumor Activities}

\author{
Li, Yaoyao Shen, Yuemao* \\ (Schoole of Pharmaceutical Sciences, Shandong Univercity, Jinan 250012)
}

\begin{abstract}
The discovery of new anti-tumor drugs is a pressing and continual need for human health due to the constant emergence of drug resistant tumor cell lines, and the rising occurrence of cancer. In recent years, researchers are paying more and more attention to the chemical components of microorganisms isolated from specific habitats and their bioactivities. Our group isolated a large number of new bioactive natural products, most with complex structures, from endophytes of medicinal plants and marine microbes. Moreover, the antitumor mechnisms and targets of two classes of compounds were investigated. Our results indicated that these special microbes are of great potential for new bioactive compounds and deserve further explorations.
\end{abstract}

Keywords endophytes of medicinal plant; marine microbes; natural product; anti-tumor action

在人类发展进程中, 疾病始终威胁着人类的生存与 繁衍, 从天然产物的药用价值被发现以来, 其在药物研 制领域就一直发挥着不可替代的作用 ${ }^{[1]}$. 目前, 尽管我 们已经拥有了许多治疗常规疾病的药物; 但是, 随着耐 药菌和新型致病菌的不断出现, 以及尚无有效治疗药物 的重大疾病(如心血管疾病、癌症等)发病率的持续攀升, 使得新结构和新作用机制药物的研发在今天显得无为 迫切. 一直以来微生物天然产物都是重要的药物来源, 由于传统研究方法重复发现天然产物的机率不断增加, 研究者们逐渐将目光投向了研究相对较少的特异生境 微生物, 如动物及植物等生物体内部(即通常所说的内 生菌)、海洋微生物和极端环境微生物(高盐碱度、超高 温、超低温等环境)等. 由于特异生境微生物的生长环境 特殊, 其产生的次级代谢产物往往具有复杂多样的结构
和广泛的生理活性, 近年来受到越来越多研究者的青 棟. 本文将主要介绍本课题组在药用植物内生菌和海洋 微生物的活性成分及其生物功能方面取得的相关研究 进展.

\section{1 药用植物内生菌是新活性化学成分的重要来 源}

植物内生菌是指那些生命过程的某一段时期或全 部生活在植物的健康组织间隙或者内部，并且对植物本 身没有引起明显病害症状的细菌(包括放线菌)或真菌. 研究显示, 植物内生菌不仅参与宿主植物代谢产物的合 成与转化, 还能够产生新的活性物质, 参与植物对外界 环境胁迫的应答 ${ }^{[2]}$. 基于 “药用植物内生菌在长期与宿 主的协同进化中可能发生一些基因的水平转移，因而产

*E-mail: yshen@sdu.edu.cn

Received April 1, 2013; revised May 6, 2013; published online May 8, 2013.

Project supported by the National Natural Science Foundation of China (Nos. 81273384, 31028019, 90913024, 30500632, 30430020, 30325044, 30070007). 国家自然科学基金(Nos. 81273384, 31028019, 90913024, 30500632, 30430020, 30325044, 30070007)资助项目. 
生与宿主相似或者相同的生物活性物质” 的假说, 以及 药用植物为内生菌提供了特别的生态环境, 其内生菌活 性成分的研究受到广泛关注.

本课题组也是鉴于上述原因, 自 1998 年开展药用 植物云南美登木和滑桃树(产生强抗肿瘤活性化合物美 登木素)内生放线菌的分离及活性成分研究, 并从中获

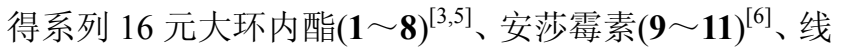

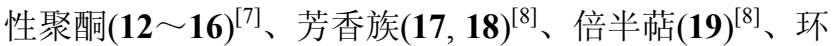
肽 $(20)^{[9]}$ 和生物碱 $(\mathbf{2 1})^{[10]}$ 类化合物. 其中, 化合物 $\mathbf{1}$ 同时 具有显著的抗真菌活性 ${ }^{[5]}$.

与此同时，以 “高等植物中的美登木素 I 型聚酮骨 架是由植物内生菌产生的” 这一假说为前提, 我们尝试 通过不依赖于纯培养的宏基因组学(metagenomics)研究 方法, 构建了滑桃树茎皮内生菌的宏基因组文库(约含 $1.77 \times 10^{6}$ 个包装颗粒, 平均插入片段约为 $\left.34.5 \mathrm{~kb}\right)^{[11]}$, 并从该文库中直接笁选获得滑桃树中可能的美登木素 生物合成基因簇, 进一步的表达与功能鉴定工作目前正
在进行中, 可能为高等植物美登木素的微生物起源提供 直接证据.

此外, 我们还开展了药用植物内生真菌的分离和活 性成分研究，包括美登木、南方红豆杉、长叶榧(红豆杉 科)、喜树、粗榧和番荔枝等. 其中，红豆杉产生的紫杉 醇和喜树产生的喜树碱都是临床上常用的抗肿瘤药物, 可治疗卵巢癌、乳腺癌和肺癌等多种癌症; 粗棑产生的 多种三尖杉酯碱，对治疗白血病及淋巴肉瘤疗效显著; 哥伦比亚番荔枝因具有多种生物活性而被当地人作为 药物广泛使用, 可用于治疗黑热病、昏睡病和㾏疾 ${ }^{[12]}$. 从这些珍贵药用植物的内生真菌中, 我们获得了结构类 型丰富多样的天然产物, 包括 10 元和 12 元大环内酯 $(\mathbf{2 2} \sim 32)^{[13 \sim 15]}$ 、三萜 $(33 \sim 35)^{[16]}$ 、二萜 $(36 \sim 50)^{[17,18]}$ 、倍

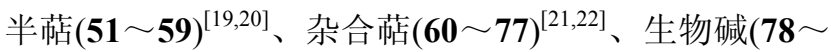
84) $)^{[15,23]}$ 和芳香族化合物 $(\mathbf{8 5} \sim 95)^{[15,18,23 \sim 25]}$. 其中，化合 物 41 对白色假丝酵母具有一定的抑制作用 ${ }^{[17]}$.

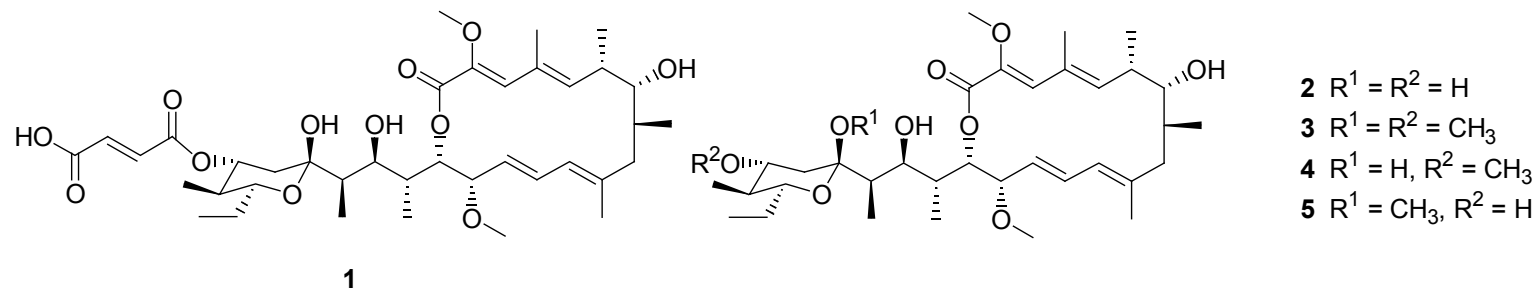

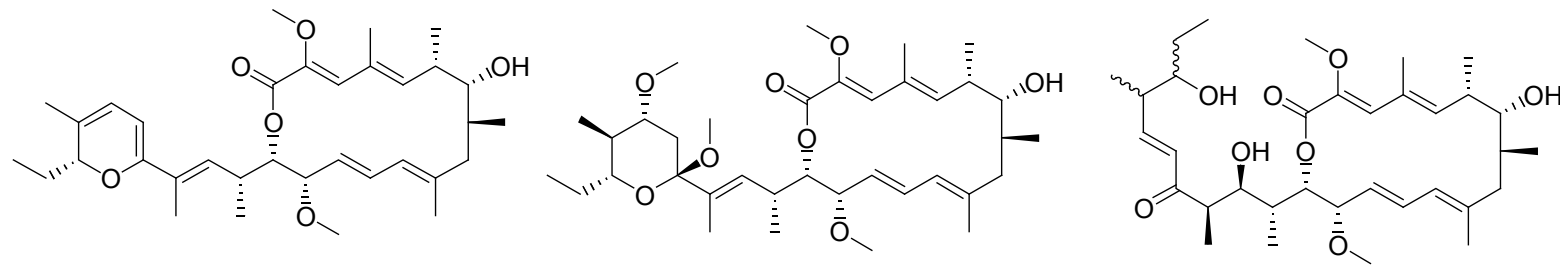

6

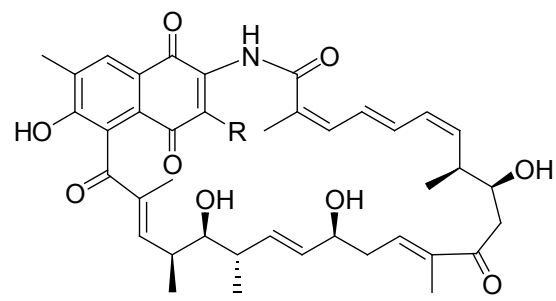

$9 \mathrm{R}=\mathrm{Cl}$ Naphthomycin A

$10 \mathrm{R}=\mathrm{H}$ Naphthomycin $\mathrm{E}$<smiles>C[C@H](OC(=O)c1cccc(NC=O)c1O)[C@@H](O)CC(N)=O</smiles>

17 Streptomyceamide A<smiles>C[C@@H]1OC(=O)c2cccc(NC=O)c2OC1=O</smiles>

18 Streptomyceamide B

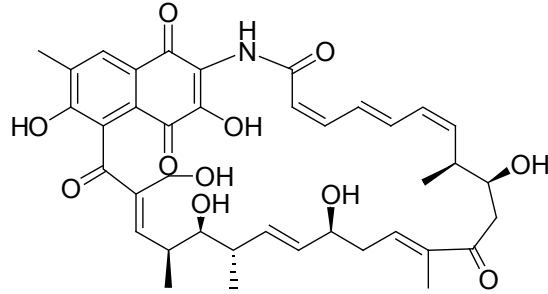

11 Naphthomycin K

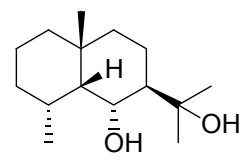

19

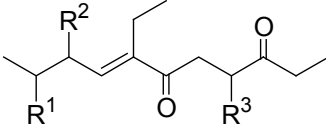

$12 R^{1}=R^{3}=H, R^{2}=O H$ $13 \mathrm{R}^{1}=\mathrm{OH}, \mathrm{R}^{2}=\mathrm{R}^{3}=\mathrm{H}$ $14 \mathrm{R}^{1}=\mathrm{R}^{2}=\mathrm{H}, \mathrm{R}^{3}=\mathrm{OH}$ $15 \mathrm{R}^{1}=\mathrm{H}, \mathrm{R}^{2}=\mathrm{R}^{3}=\mathrm{OH}$ $16 \mathrm{R}^{1}=\mathrm{R}^{3}=\mathrm{OH}, \mathrm{R}^{2}=\mathrm{H}$ 
<smiles>[R]C(=O)CCC(=O)OC(C)CC([R])CCCC([R])CC</smiles>

$22 \mathrm{R}^{1}=\mathrm{H}, \mathrm{R}^{2}=\mathrm{OH}$ $23 \mathrm{R}^{1}=\mathrm{OH}, \mathrm{R}^{2}=\mathrm{H}$<smiles>CC1CCCCCCC(O)C(O)CC(=O)O1</smiles>

24<smiles>CCCCCCC(O)C(O)/C=C\C(=O)OC(=O)/C=C/C(O)C(O)CCCCCC(C)OC</smiles>

$\mathrm{OH}$

25 Cladospolide A 26 Cladospolide B<smiles>CC(O)C[C@@H](O)[C@H](O)C=CC1OC1C(=O)O</smiles>

$27 \mathrm{R}=\mathrm{Ac}$ $28 \mathrm{R}=\mathrm{H}$<smiles>CC1C[C@@H](O)C(O)C2OC2C2OC2C1=O</smiles>

$29 \mathrm{R}=\mathrm{Ac}$ $30 \mathrm{R}=\mathrm{H}$<smiles>CC(C)C[C@H](O)[C@@H](O)/C=C/C=C\C(=O)O</smiles>

31

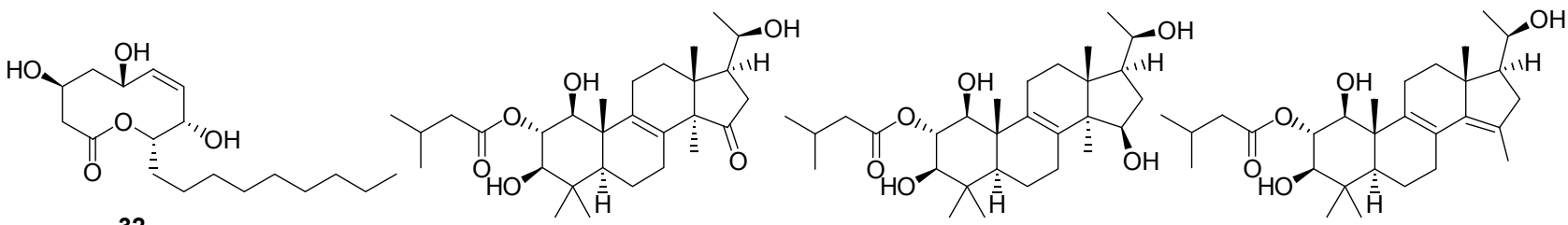

33 Xylariacin $\mathrm{A}$

34 Xylariacin B

35 Xylariacin C

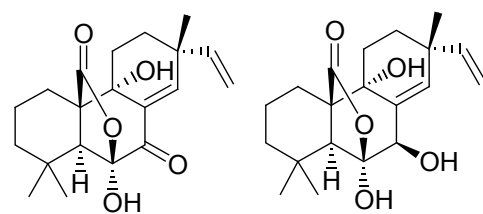

36 Sphaeropsidin A 37 Sphaeropsidin B

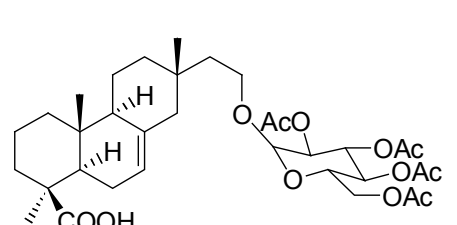

38<smiles></smiles>

39 Botryosphaerin A<smiles>C[C@]12CCC[C@@]3(C)C(=O)O[C@H](C=C4COC(=O)C[C@@H]41)C[C@H]23</smiles>

40<smiles>C[C@@]12CCC[C@@]3(C)C4=CC(=O)OCC4=C[C@H](C[C@@]13C)OC2=O</smiles>

41 CJ-14445<smiles>CC1(C(=O)O)CCCC[C@@]12CCC1COC(=O)C[C@H]1C2</smiles>

42 Acrostalidic acid<smiles>CC12CCC[C@@](C)(C(=O)O)C1C=C[C@]1(CO)OC(=O)C[C@@H]21</smiles>

43 Botryosphaerin B<smiles>CC1(O)CC[C@H]2C(=C1C(=O)O)CC[C@]1(C)[C@@H](C(=O)O)CCC[C@@]21C</smiles>

44 Botryosphaerin E<smiles>[R]C1=C2CC[C@]3(C)[C@@](C)(C(=O)O)CCC[C@]3(C)[C@H]2CC1</smiles>

$45 \mathrm{R}=\mathrm{COOH}$ Agathic acid $46 \mathrm{R}=\mathrm{CH}_{2} \mathrm{OH}$ Isocupressic acid<smiles>[R]CC1C(=C)C([R])CC2(C)C([R4])CCCC12C</smiles>

$47 \mathrm{R}^{1}=\mathrm{COOH}, \mathrm{R}^{2}=\mathrm{H}, \mathrm{R}^{3}=\mathrm{CH}_{2} \mathrm{OH}$ Botryosphaerin $\mathrm{C}$ $48 \mathrm{R}^{1}=\mathrm{CH}_{2} \mathrm{OH}, \mathrm{R}^{2}=\mathrm{H}, \mathrm{R}^{3}=\mathrm{COOH}$ Botryosphaerin $\mathrm{D}$ $49 \mathrm{R}^{1}=\mathrm{COOH}, \mathrm{R}^{2}=\mathrm{H}, \mathrm{R}^{3}=\mathrm{COOH}$ Acrostalic acid $50 \mathrm{R}^{1}=\mathrm{COOH}, \mathrm{R}^{2}=\mathrm{OH}, \mathrm{R}^{3}=\mathrm{COOH}$ LL-Z1271<smiles>Cc1c(C(O)(CO)CO)ccc2c1C(C)CC=C2</smiles>
51 Tuberculariol A<smiles>C=C(CO)c1ccc2c(c1C)C(C)C[C@@H](O)[C@H]2O</smiles>

52 Tuberculariol B<smiles>C=C(CO)[C@H]1CC2(C)C(C)CCCC2(O)C(O)C1O</smiles>

53 Tuberculariol C<smiles>[R]CC(=C)[C@]12O[C@@]1(C)[C@@H](C)CC[C@@H]2O</smiles>

$54 \mathrm{R}=\mathrm{OH}$ Xylarenones $\mathrm{A}$ $55 \mathrm{R}=\mathrm{OAc}$ Xylarenones $\mathrm{B}$<smiles>CC(C(=O)O)=C1CC(C)(C)CC2C1CCC2(C)O</smiles>

56 Xylarenic acid

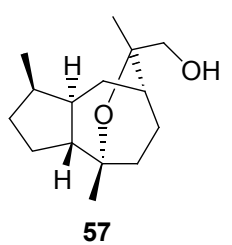

57

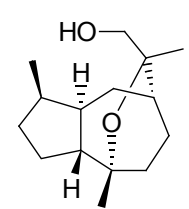

58

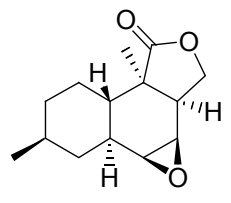

65 Oblongolide $P$

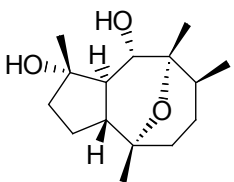

59

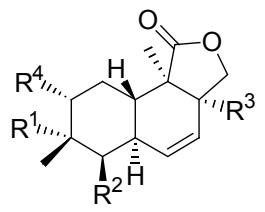

$60 \mathrm{R}^{1}=\mathrm{R}^{3}=\mathrm{OH}, \mathrm{R}^{2}=\mathrm{R}^{4}=\mathrm{H}$ Oblongolide $\mathrm{B}$ $61 R^{1}=R^{2}=R^{4}=H, R^{3}=O H$ Oblongolide $C$ $62 \mathrm{R}^{1}=\mathrm{R}^{2}=\mathrm{R}^{3}=\mathrm{H}, \mathrm{R}^{4}=\mathrm{OH}$ Oblongolide $\mathrm{H}$ $63 \mathrm{R}^{1}=\mathrm{R}^{2}=\mathrm{H}, \mathrm{R}^{3}=\mathrm{R}^{4}=\mathrm{OH}$ Oblongolide $\mathrm{N}$ $64 R^{1}=R^{3}=R^{4}=H, R^{2}=O H$ Oblongolide $O$

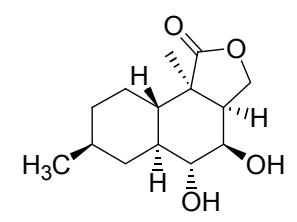

66 Oblongolide $Q$ 
<smiles>CC1CC[C@H]2C(C=C[C@](O)(CO)[C@@]2(C)C(=O)O)C1</smiles>

67 Oblongolide R

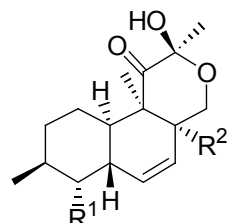

$68 \mathrm{R}^{1}=\mathrm{R}^{2}=\mathrm{H}$ Decumbenone $\mathrm{C}$

$69 \mathrm{R}^{1}=\mathrm{H}, \mathrm{R}^{2}=\mathrm{OH}$ Decumbenone $\mathrm{D}$

$70 \mathrm{R}^{1}=\mathrm{OH}, \mathrm{R}^{2}=\mathrm{H}$ Decumbenone $\mathrm{E}$<smiles>[R]C(C)CCCC(C)C1=CC[C@@H]2OC3=C(C[C@]12[R1])C(=O)C(O)CC3</smiles>

$71 \mathrm{R}^{1}=\mathrm{H}, \mathrm{R}^{2}=\mathrm{COOH}$

$72 \mathrm{R}^{1}=\mathrm{H}, \mathrm{R}^{2}=\mathrm{CH}_{2} \mathrm{OAC}$

$73 \mathrm{R}^{1}=\mathrm{H}, \mathrm{R}^{2}=\mathrm{CH}_{2} \mathrm{OH}$

$74 \mathrm{R}^{1}=\mathrm{OH}, \mathrm{R}^{2}=\mathrm{CH}_{2} \mathrm{OH}$<smiles>[R]CC(C)=CCCC(C)C1=CC[C@H]2OC3=C(C[C@H]12)C(=O)[C@H](O)CC3</smiles>

$75 \mathrm{R}=\mathrm{Ac}$

$76 \mathrm{R}=\mathrm{H}$<smiles>CC(CCC(=O)O)C1=CC[C@H]2OC3=C(C[C@H]12)C(=O)[C@H](O)CC3</smiles>

77<smiles>COc1ccc2c3c([nH]c2c1)[C@@H](O)[C@@]1(O)C(=O)N2CCC[C@H]2C(=O)N1[C@H]3C=C(C)C</smiles>

78 Cyclotryprostatin A<smiles>COc1ccc2c3c([nH]c2c1)[C@@H](CC(C)(C)O)N1C(=O)[C@]2(C)CCCN2C(=O)[C@]31O</smiles>

79<smiles>COc1ccc2c3c([nH]c2c1)C(CC(C)(C)O)N1C(=O)C(C)(C)N(C)C(=O)[C@]31O</smiles>

80

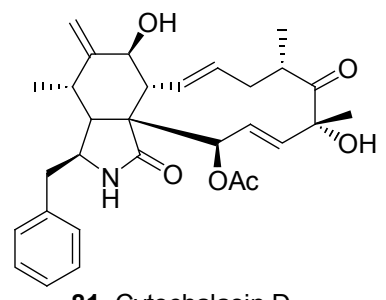

81 Cytochalasin D

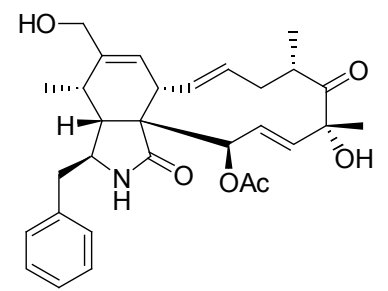

82 12-Hydroxyzygosporin G<smiles>C=C1C(O)[C@H](C=CC[C@@H](C)C(=O)C(C)C=CC(C)OC(C)=O)C2(C(=O)NC(Cc3ccccc3)[C@@H]2C)[C@@H]1C</smiles>

83 Zygosporin $\mathrm{E}$

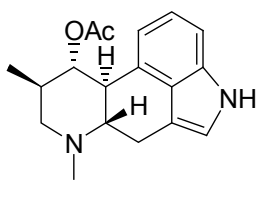

84 Isofumigaclavine A

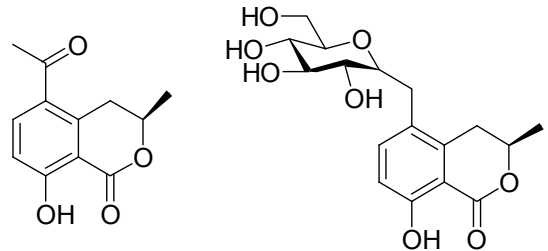

86

85 5-Methyoxycarbonylmellein<smiles>CC1OC(=O)c2c(O)cccc2C1O</smiles>

87 4-Hydroxymellein<smiles>CC1OC(=O)c2c(ccc(Cl)c2O)[C@H]1O</smiles>

88<smiles>CC1Cc2c(O)cc(Cl)c(O)c2C(=O)O1</smiles>

89<smiles>O=C1CC(O)Cc2cc(O)c(Cl)c(O)c21</smiles>

90<smiles>COc1cc(O)cc2c1C(=O)c1c(O)cc(C)cc1C2=O</smiles>

91 Methylemodin<smiles>COC1=CC(=O)C=C(C(=O)O)C12Oc1cc(C)cc(OC)c1C2=O</smiles>

92 Trypacidin<smiles>COC(=O)c1cc(O)cc(OC)c1C(=O)c1c(O)cc(C)cc1OC</smiles>

93 1, 2-seco-Trypacidin<smiles>COC(=O)c1cc(OC)c2c(=O)c3cc(O)cc(OC)c3oc3c(OC)cc(C)cc3c(=O)oc2c1</smiles>

\section{2 海洋生物共附生菌是新活性化学成分的重要} 来源

海洋是地球万物的起源之地, 从海洋中最原始生命 出现至今已有 40 多亿年历史. 在这漫长的演化过程中 海洋孕育了丰富的生物资源, 成为地球上物种资源最丰 富的领域 ${ }^{[26]}$. 此外, 由于海洋环境的特殊性 (高压、低 氧、高盐、闭光等), 海洋生物间的生存竞争更为激烈, 为适应严酷的生存环境, 许多海洋生物进化出与陆生生 物不同的代谢机制和防御机制, 这也意味着我们很可能 从中发现新的物种和结构新颖、生物活性显著的次级代
谢产物 ${ }^{[27]}$. 因此, 海洋生物共附生菌活性成分的研究备 受瞩目.

本课题组在对海洋共附生放线菌的分离和其次级 代谢产物研究中, 采取定向笁选和分离具有合成安莎类 化合物潜能的海洋放线菌，以期从中获得全新结构类型 的安莎类化合物. 众所周知, 安莎是一类重要的抗生素, 主要分布于放线菌中. 虽然目前仅发现了 200 余个, 但 是大部分具有广泛的生理活性、成药率高. 其中, 最具 代表性的格尔德霉素和美登木素的衍生物已用于肿瘤 治疗，利福霉素的衍生物利福平是治疗结核的一线药 
物. 安莎类抗生素属于结构特征显著的 I 型聚酮, 已发 现的结构可以分为 5 酮、7 酮、8 酮、9 酮、11 酮和 14 酮. 根据 I 型聚酮类化合物的生物合成特点, 我们有理 由推测, 自然界中还可能存在 6 酮、10 酮、12 酮和 13 酮等类型的安莎类化合物. 因此, 寻找新资源发掘新颖 安莎类化合物，对于新药创制具有重要意义.

近年来, 我们从 688 株海洋放线菌中篎选得到了 21 株具有合成安莎霉素潜能的阳性菌株. 通过对 21 株阳 性菌株的培养基篮选、发酵条件优化和定向分离, 已经 获得部分阳性菌株代谢产生的安莎霉素类抗生素 $(\mathbf{9 6}$ ～ 112), 以及系列活性新安莎(数据未给出).

同时, 我们也开展了潮间带红树植物内生真菌的分 离和代谢产物研究. 红树林生态系统处于陆地和海洋的 动态交界面, 受周期性海水浸渍, 使其形成了既不同于 陆地也不同于海洋的独特生态系统. 因此, 与红树植物 协同进化的内生菌很可能具有独特的次生代谢途径有 待我们发掘. 通过对多株红树内生真菌代谢产物的系统
分离，我们获得了一系列具有显著生理活性的天然产

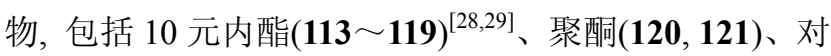

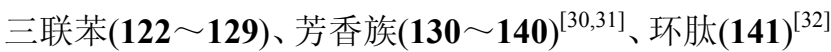
生物碱 $(142,143)^{[29]}$ 和苯并呋喃酮 $(144)^{[28]}$. 其中, 化合 物 113,114 和 144 对白色假丝酵母的最低抑制浓度 (MIC) 分别为 10,5 和 $10 \mu \mathrm{g} / \mathrm{mL}$; 化合物 144 对枯草芽孢 杆菌的最小抑制浓度为 $1.25 \mu \mathrm{g} / \mathrm{mL}^{[28]}$; 化合物 $\mathbf{1 2 0}$ 129 具有显著的抗肿瘤活性和新颖的作用机制 ${ }^{[33,34]}$.

\section{3 抗肿瘤活性研究}

对上述分离得到的化合物进行抗肿瘤活性测定，发 现许多具有较高细胞毒性的活性化合物. 其中，化合物 1 和 5 对人肺癌细胞 A-549 的 IC 50 分别为 $<0.01$ 和 0.01 $\mu \mathrm{mol} / \mathrm{L}^{[4,5]}$, 化合物 $\mathbf{2} \sim \mathbf{4}, \mathbf{7}$ 和 8 对人乳腺癌细胞 MDAMB-435 的 IC50 分别为 4.2, 5.5, 4.5, 3.8 和 $11.4 \mu \mathrm{mol} / \mathrm{L}^{[3]}$; 化合物 11 对白血病细胞 $\mathrm{P} 388$ 的 $\mathrm{IC}_{50}$ 为 $0.07 \mu \mathrm{mol} / \mathrm{L}^{[6]}$;

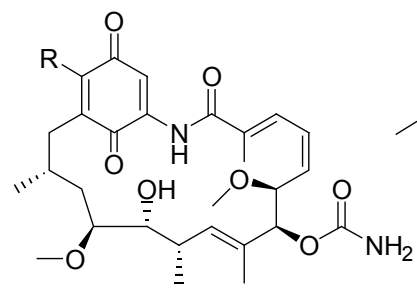

$96 \mathrm{R}=\mathrm{OMe}$ Geldanamycin $97 \mathrm{R}=\mathrm{OH}$ 17-O-Methylgeldanamycin $98 \mathrm{R}=\mathrm{H}$ Herbimycin $\mathrm{B}$

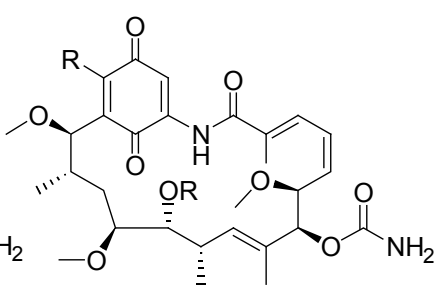

$99 \mathrm{R}=\mathrm{CH}_{3}$ Herbimycin $\mathrm{A}$ $100 \mathrm{R}=\mathrm{H}$ Herbimycin $\mathrm{C}$

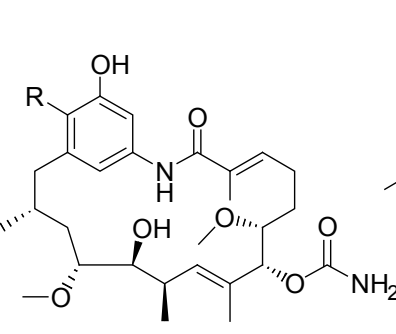

$101 \mathrm{R}=\mathrm{OMe}$ Reblastatin $102 \mathrm{R}=\mathrm{OH}$ 17-O-Methylreblastatin $103 \mathrm{R}=\mathrm{H}$ Autolytimycin

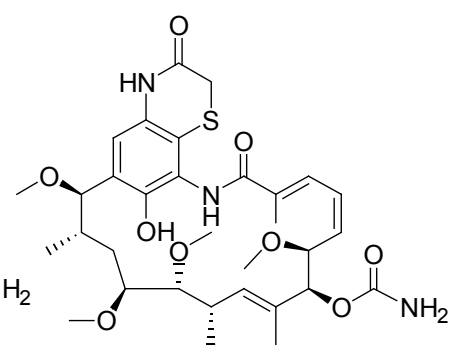

104 Heronamycin A

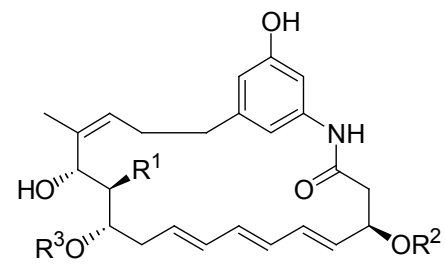

$105 R^{1}=R^{2}=$ Me, $R^{3}=a$ Trienomycin $A$ $106 R^{1}=R^{2}=R^{3}=H$ Demethyltrienomycinol $107 \mathrm{R}^{1}=\mathrm{R}^{2}=\mathrm{H}, \mathrm{R}^{3}=\mathbf{a}$ Demethyltrienomycin $\mathrm{A}$<smiles>[R6]C(C/C=C/C=C/C=C/C(CO)OC)[C@H](O)[C@H](C)/C(C)=C\CCc1c(O)c(NC(=O)CC(C)O)cc2c1SCC(=O)N2</smiles>

$108 \mathrm{R}=\mathrm{a}$ Thiazinothrienomycin $\mathrm{E} \quad 109 \mathrm{R}=\mathrm{a}$ Thiazinotrienomycin $\mathrm{G}$

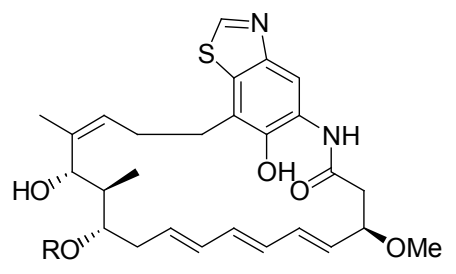<smiles>CC(=O)C(C)NC(=O)C1CCCCC1</smiles>

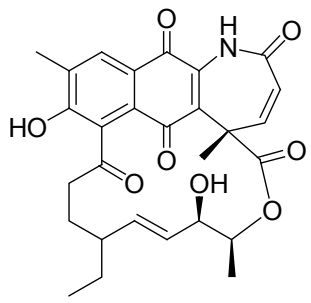

110 Hygrocin B

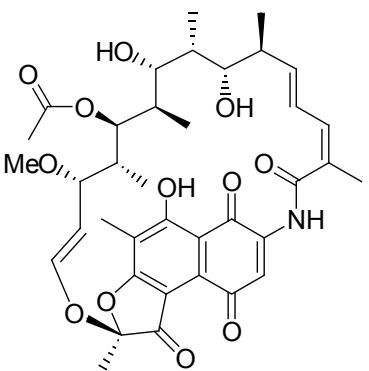

111 Rifamycin

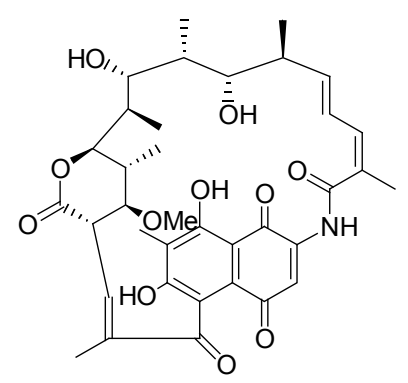

112 27-O-Methylrifamycin Z 


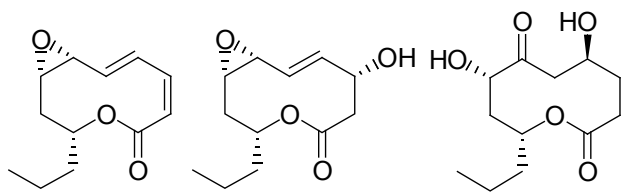

113 Phomolide A Phomolide B
115 Phomolide D<smiles>CCC(O)[C@H](C[C@@H](O)[C@@H](O)/C=C\C=C/C(=O)O)OC</smiles>

116 Phomolide E<smiles>CC(O)C[C@H](C[C@@H](O)[18F])OC(=O)/C=C\C=C/[C@H](O)[In]</smiles>

Phomolide F<smiles></smiles>

119

Phomolide $\mathrm{H}$<smiles>CC(=O)O[C@H]1C=CC(=O)O[C@@H]1[C@H]1C2C=CC=C(O2)[C@H]1C</smiles>

120

Mycoepoxydiene

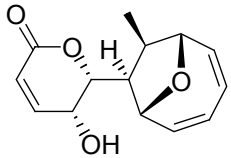

121

Deacetylmycoepoxydiene<smiles>[R]c1ccc(-c2cc(OC)c(-c3ccc(O)c([R])c3)c(O)c2OC)cc1[R]</smiles>

$122 \mathrm{R}^{1}=\mathrm{R}^{2}=\mathrm{H} ; \mathrm{R}^{3}=\mathrm{OH}$

$123 R^{1}=R^{3}=H ; R^{2}=O H$

$124 R^{1}=\mathrm{H} ; R^{2}=\mathrm{R}^{3}=\mathrm{OH}$

$125 \mathrm{R}^{1}=\mathrm{R}^{2}=\mathrm{R}^{3}=\mathrm{OH}$

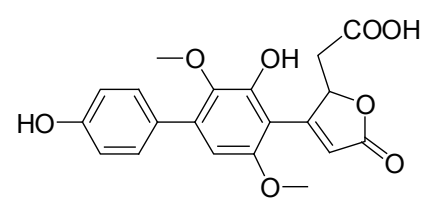

126<smiles>COc1cc(C2=CC(=O)OC2CC(=O)O)c(OC)c(O)c1C1=CC(=O)OC1CC(=O)O</smiles>

127<smiles>[R]c1cc(-c2cc(OC)c3c(oc4cc(O)c(O)cc43)c2OC)ccc1O</smiles>

$128 \mathrm{R}=\mathrm{H}$<smiles>[R]CC([R])c1c(OC)cc2c(c1O)C(=O)C[C@@H](I)[C@H]2O</smiles>
$130 \mathrm{R}^{1}=\mathrm{R}^{2}=\mathrm{OH}, \mathrm{R}^{3}=\mathrm{H}$ $131 \mathrm{R}^{1}=\mathrm{R}^{3}=\mathrm{OH}, \mathrm{R}^{2}=\mathrm{H}$ $132 \mathrm{R}^{1}=\mathrm{OH}, \mathrm{R}^{2}=\mathrm{OMe}, \mathrm{R}^{3}=\mathrm{H}$ $133 \mathrm{R}^{1}=\mathrm{OH}, \mathrm{R}^{2}=\mathrm{R}^{3}=\mathrm{H}$ $134 R^{1}=R^{2}=R^{3}=H$<smiles>[R]C(C)c1c(OC)cc2c(c1O)C(=O)C=C(OC)C2=O</smiles>

$135 \mathrm{R}=\mathrm{H}$ $136 \mathrm{R}=\mathrm{OAC}$

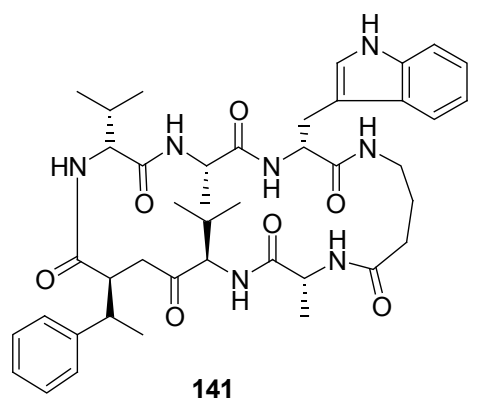<smiles>COc1cccc(Oc2c(C=O)cc(C)cc2C=O)c1C(=O)O</smiles>

137<smiles>COc1cccc2c1C(=O)Oc1cc(CO)cc(O)c1O2</smiles>

138<smiles>[R]c1cc(Cl)c(O)c(-c2oc3c(OC)c(OC)cc(O)c3c(=O)c2OC)c1</smiles>

$139 \mathrm{R}=\mathrm{H}$ $140 \mathrm{R}=\mathrm{OH}$
化合物 76 在 $16 \mu \mathrm{g} / \mathrm{mL}$ 浓度下对人肺癌细胞 A-549 和急 性早幼粒白血病 HL-60 的抑制率分别为 $88.6 \%$ 和 $98.7 \%{ }^{[21]}$. 我们对其中 2 类化合物开展了较深入的活性 机理研究.

\subsection{Mycoepoxydiene 同时激活 p53 和抑制 NF-kB 信 号途径}

随着癌症发生发展机制研究的深入, 针对癌症相关 信号转导通路的靶向治疗研究得到迅速发展, 并提供了
不少临床用药. 抑癌基因 p53 和核转录因子 NF- $\mathrm{KB}$ 不仅 在许多体内生理过程中起着重要作用, 在肿瘤的发生和 发展中也发挥着非常关键的作用, p53 的基因突变或者 功能性失活以及 NF-KB 的过度激活都会导致多种常见 癌症 ${ }^{[35]}$. 因此, 已有不少作用于 p53 信号转导通路或者 NF-KB 信号转导通路的小分子被开发并运用于临床, 不 过仅有少数能够同时作用于这两条信号传导通路，如： R-roscovitine ${ }^{[36]}$, flavopiridol ${ }^{[37]}$, 9-aminoacridine (9AA) derivatives $^{[38]}$, nutlin- $3^{[39]}$ 和 cisplatin $^{[40]}$. 
我们的研究表明, Mycoepoxydiene (MED, 120)是一 个能够激活肿瘤细胞 MCF-7 内 p53 信号传导通路, 同时 抑制 NF-KB 信号转导通路，具有良好应用潜力的天然产 物(图 1). 在 MED 作用下, MCF-7 细胞内的活性氧(ROS) 水平迅速上升, 导致基因组 DNA 出现双链断裂损伤 (Double-stranded Breaks). DNA 的损伤启动 ATM-Chk2p53 修复机制, 但是由于损伤严重, 细胞基因组 DNA 得 不到完全修复, 细胞走向调亡. p53 分子在 MED 引起的 调亡中起着关键作用, 但是 p 53 基因的敲除并不能完全 抑制 MED 引起的细胞凋亡, 这提示我们 MED 引起的细 胞调亡过程中可能有其他信号转导途径的参与. 由于在 细胞增殖的过程中具有重要作用的 NF-KB 对细胞内 ROS 水平的变化也较为敏感, 因此, 我们检测了 MED 对细胞内 NF-kB 信号转导通路的影响. 实验结果表明, MED 可以抑制由 TNF- $\alpha$ 所激活的 NF-KB 信号转导途径, 其机制为 MED 促进细胞内 ROS 水平增加后, 热休克蛋 白 Hsp27 Ser-78/82 发生磷酸化, 因而促进 Hsp27 与 IKK $\gamma$ 的相互作用, 使得 RelA 的入核转运受到抑制. 但 是, 我们并没有发现 MED 所引起的 p53 信号转导通路 激活和 NF- $\mathrm{KB}$ 信号转导通路抑制之间存在着 cross-talk. MED 的特殊作用机制显示其具有良好的临床应用潜力, 值得开展更深入的研究.

\section{2 对三联苯是新型拓扑异构酶抑制剂}

拓扑异构酶(topoisomerase) 是广泛存在于真核和原 核生物体内的基本酶之一, 调节 DNA 空间结构动态变 化, 参与 DNA 复制、转录、重组和修复等生理过程, 在 细胞生命过程中起着重要的作用 ${ }^{[41]}$. 拓扑异构酶主要 包括两类酶: 拓扑异构酶 I (topoisomerase I, Topo I) 和 DNA 拓扑异构酶 II (topoisomerase II, Topo II). 由于肿 瘤细胞具有快速增殖的特性, 其拓扑异构酶的含量及活
性远远高于正常体细胞，抑制拓扑异构酶活性就能起到 阻止肿瘤细胞快速增殖, 进而杀死肿瘤细胞的作 用 ${ }^{[22,43]}$. 因此，以 DNA 拓扑异构酶为靶点的抗肿瘤药 物一直是抗肿瘤药物研发的热点之一 ${ }^{[44]}$.

对三联苯是一类主要分布于地衣和真菌的重要天 然产物, 文献报道该类化合物有多种生理活性 ${ }^{[45]}$, 包括 抗肿瘤活性 ${ }^{[46-49]}$. 前期, 我们从海洋真菌 AF119 菌株中 分离得到 8 个对三联苯类化合物( $p$-terphenyls, 122 129), 对多种肿瘤细胞增殖均有较强的抑制作用. 为系 统研究该类化合物抗肿瘤作用的构效关系, 我们设计合 成了一系列新的对三联苯衍生物 $(\mathbf{H 1} \sim \mathbf{H 8})$. 活性研究 结果表明, 化合物 $\mathbf{H 1} \sim \mathbf{H 8}$ 具有更强的抗肿瘤活性, 且 其活性与抑制 TOPII $\alpha$ 有关. 其中, 活性最强的化合物 H2 通过诱导 MDA-MB-435 细胞 G2/M 期停滞和 caspase 依赖性细胞调亡，显著抑制细胞增殖， $\mathrm{IC}_{50}$ 为 0.39 $\mu \mathrm{mol} / \mathrm{L}$. 体外 DNA 松弛实验表明, H2 对 TOPI 和 TOPII $\alpha$ 均有较强的抑制作用. 进一步的研究表明, $\mathbf{H} 2$ 并不引起 TOP 介导的 DNA 断裂, 也不能嵌入到 DNA, 可能是新 结构类型的 TOPI 和 TOPII $\alpha$ 催化抑制剂(图 2). 此外, 我 们还发现, 结构相似的化合物 H8, 对 MDA-MB-435 细 胞的 $\mathrm{IC}_{50}$ 为 $4.1 \mu \mathrm{mol} / \mathrm{L}$, 选择性抑制 TOP II $\alpha$. 综上所述, TOP II $\alpha$ 可能是对三联苯类化合物在细胞内的一个主要 作用靶点, $\mathrm{H2}$ 和 $\mathrm{H8}$ 是设计与合成 TOP II $\alpha$ 选择性抑制 剂的先导物.

\section{4 结论}

天然产物是药物先导化合物的源头, 如何快速有效 的发现具有药物开发潜力的新活性天然产物是亟待解 决的关键问题. 特异生境微生物蕴含着丰富的天然产物 资源，它们在适应特殊生存环境的过程中进化出许多独

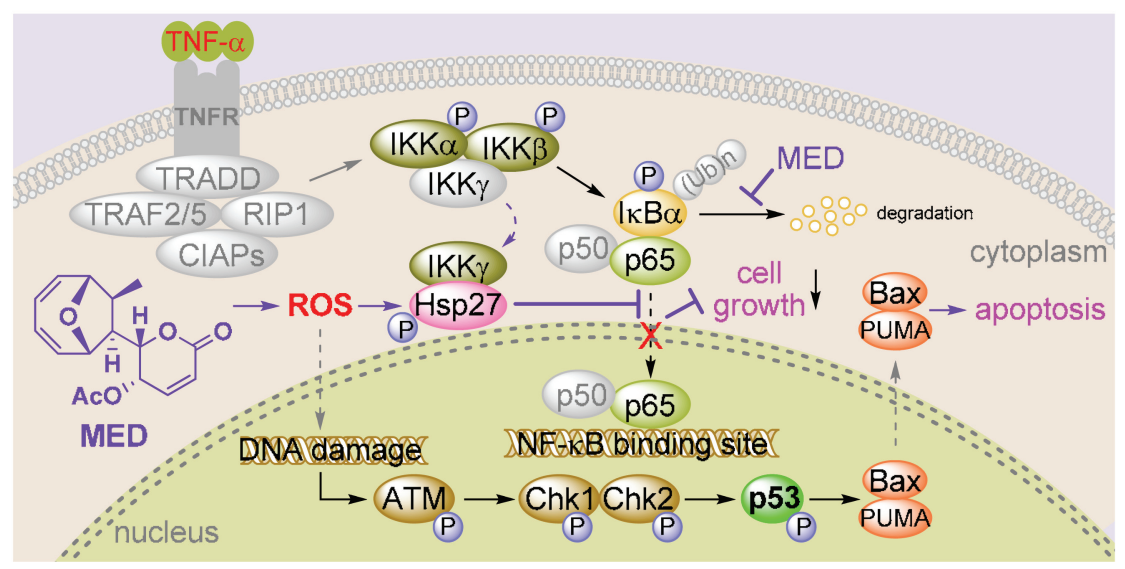

图 1 MED 通过诱导活性氧的产生在 MCF-7 细胞中同时激活 p53 和抑制 NF-KB 信号通路

Figure 1 MED simultaneously activates p53 and inhibits NF-кB signaling pathways through the production of reactive oxygen species (ROS) in MCF-7 cell 


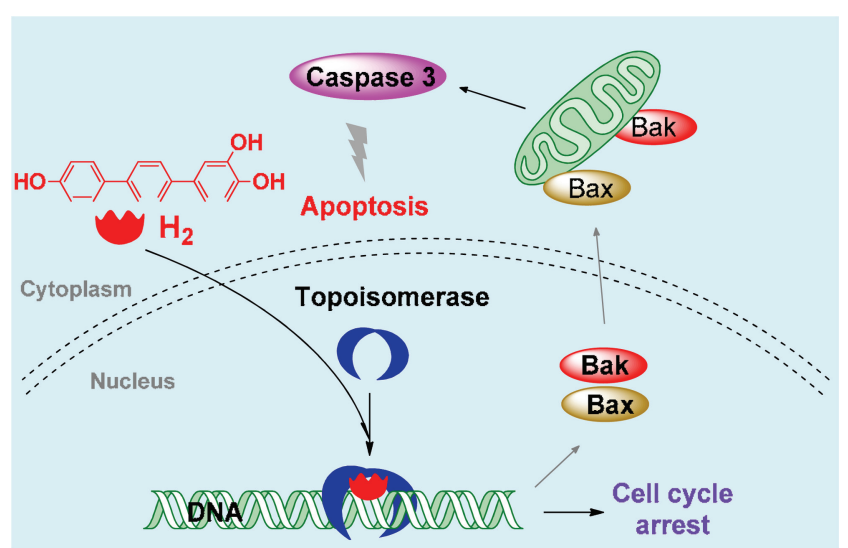

图 $2 \mathrm{H2}$ 的化学结构与生物学功能

Figure 2 The chemical structure and biological functions of $\mathbf{H 2}$ 特的次生代谢途径, 从而成为新活性天然产物的重要来 源. 本课题组对药物植物内生菌和海洋微生物天然产物 的发掘, 以及对 2 个抗肿瘤活性化合物的作用机制研究, 进一步展示了特异生境微生物代谢产物的结构新颖性、 多样性和独特的活性作用机制. 在后续研究中, 一方面 需要继续挖掘特异生境中蕴藏的新颖微生物资源, 另一 方面需要通过多种手段(如培养优化、菌株诱变和遗传 操作等) 充分释放这些新颖微生物的次生代谢潜能, 并 同时开展新活性化合物的作用机制研究，从而为药物先 导化合物的发现奠定基础.

\section{作者简介}

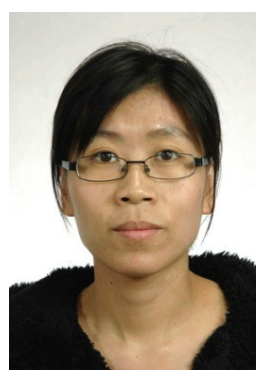

李瑶瑶, 博士, 讲师; 1982 年 11 月出生于吉林. 2005 年厦门大学生命科学学院本科毕业(导师: 沈月毛 教授). 2010 年厦门大学生命科学学院博士毕业, 主要开 展真菌萜类化合物及其生物合成研究(导师: 沈月毛教 授). 2008 2009 年美国内布拉斯加州立大学林肯分校 访问学习, 参与了多个活性化合物的生物合成研究(合 作导师: 杜良成教授). 2010 2012 年在山东大学药学院 从事博士后研究(合作导师: 娄红祥教授). 2012 年获聘 山东大学药学院讲师, 主要从事产酶溶杆菌的活性成分 及其生物合成研究.

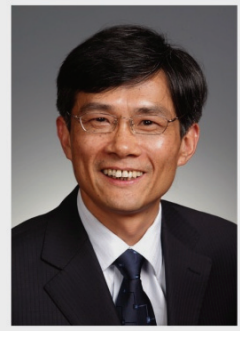

沈月毛，博士，教授; 1965 年 2 月出生于安徽; 从事 天然产物化学生物学和微生物药物研究. 自 1986 年于 安徽师范大学化学系毕业后, 在中国科学院昆明植物研 究所获得硕士和博士学位, 期间主要从事植物化学与天 然药物开发研究; 1995 年留学美国华盛顿大学(西雅图), 师从 Floss 教授, 从事安莎类抗生素的生物合成研究; 1998 年回国后致力于微生物活性天然产物的发现、生物 功能、作用机制、生物合成及其调控机制的研究. 在 Nat. Chem. Biol., Proc. Natl. Acad. Sci. USA 和 J. Am. Chem. Soc. 等刊物发表论文 100 余篇; 申请新药发明专利 42 件, 获授权 22 件; 2003 年获国家杰出青年科学基金资助.

\section{References}

[1] Cragg, G. M.; Newman, D. J.; Snader, K. M. J. Nat. Prod. 1997, 60,52 .

[2] Saikkonen, K.; Wali, P.; Helander, M.; Faeth, S. H. Trends Plant Sci. 2004, 9, 275.

[3] Li, J.; Lu, C.; Shen, Y. J. Antibiot. (Tokyo) 2010, 63, 595.

[4] Lu, C.; Shen, Y. J. Antibiot. (Tokyo) 2004, 57, 597.

[5] Lu, C.; Shen, Y. J. Antibiot. (Tokyo) 2003, 56, 415.

[6] Lu, C.; Shen, Y. J. Antibiot. (Tokyo) 2007, 60, 649.

[7] Li, J.; Lu, C.; Shen, Y. Helv. Chim. Acta 2008, 91, 741.

[8] Zhao, P. J.; Li, G. H.; Shen, Y. M. Chem. Biodivers 2006, 3, 337.

[9] Zhao, P. J.; Wang, H. X.; Li, G. H.; Li, H. D.; Liu, J.; Shen, Y. M. Chem. Biodivers 2007, 4, 899.

[10] Zhu, N.; Zhao, P.; Kang, Q.; Shen, Y. Nat. Prod. Res. Dev. 2008 $20,395$.

[11] Wang, H. X.; Geng, Z. L.; Zeng, Y.; Shen, Y. M. Environ. Microbiol. 2008, 10, 2684.

[12] Osorio, E.; Arango, G. J.; Jimenez, N.; Alzate, F.; Ruiz, G.; Gutierrez, D.; Paco, M. A.; Gimenez, A.; Robledo, S. J. Ethnopharmacol. 2007, 111, 630.

[13] Dai, H.; Kang, Q.; Li, G.; Shen, Y. Helv. Chim. Acta 2006, 89, 527.

[14] Tan, Q.; Yan, X.; Lin, X.; Huang, Y.; Zheng, Z.; Song, S.; Lu, C.; Shen, Y. Helv. Chim. Acta 2007, 90, 1811.

[15] Xu, R.; Lu, C.; Wu, X.; Lin, C.; Zheng, Z. Chem. Nat. Compd. 2009, 45, 797.

[16] Lin, T.; Lin, X.; Lu, C.-H.; Shen, Y.-M. Helv. Chim. Acta 2011, 94, 301.

[17] Yuan, L.; Zhao, P.-J.; Ma, J.; Lu, C.-H.; Shen, Y.-M. Helv. Chim. Acta 2009, 92, 1118

[18] Li, Y.; Lu, C.; Hu, Z.; Huang, Y.; Shen, Y. Nat. Prod. Res. 2009, 23,70 .

[19] Hu, Z.-Y.; Li, Y.-Y.; Huang, Y.-J.; Su, W.-J.; Shen, Y.-M. Helv. Chim. Acta 2008, 91, 46.

[20] Xu, R.; Wang, M.-Z.; Lu, C.-H.; Zheng, Z.-H.; Shen, Y.-M. Helv. Chim. Acta 2009, 92, 1514 
[21] Yuan, L.; Zhao, P.-J.; Ma, J.; Li, G.-H.; Shen, Y.-M. Helv. Chim. Acta 2008, 91, 1588.

[22] Lin, T.; Lin, X.; Lu, C.; Hu, Z.; Huang, W.; Huang, Y.; Shen, Y. Eur. J. Org. Chem. 2009, 2009, 2975.

[23] Xue, H.; Lu, C.; Liang, L.; Shen, Y. Rec. Nat. Prod. 2012, 6, 28.

[24] Lu, C.; Huang, Y.; Shen, Y. Chin. J. Nat. Med. 2005, 3, 269.

[25] Lu, C.; Lin, X.; Shen, Y. Chem. Nat. Compd. 2008, 44, 569.

[26] Haefner, B. Drug Discovery Today 2003, 8, 536.

[27] Glaser, K. B.; Mayer, A. M. Biochem. Pharmacol. 2009, 78, 440.

[28] Du, X.; Lu, C.; Li, Y.; Zheng, Z.; Su, W.; Shen, Y. J. Antibiot. (Tokyo) 2008, 61, 250.

[29] Li, Y.; Wang, M.; Huang, Y.; Shen, Y. Mycology 2010, 1, 254.

[30] Xu, Y.-H.; Lu, C.-H.; Zheng, Z.-H.; Shen, Y.-M. Helv. Chim. Acta 2011, 94, 897.

[31] Liu, S.; Lu, C.; Huang, Y.; Shen, Y. Rec. Nat. Prod. 2012, 6, 334.

[32] Liu, S.; Shen, Y. Chem. Nat. Compd. 2011, 47, 786.

[33] Zhu, J.; Chen, Q.; Xia, X.; Mo, P.; Shen, Y.; Yu, C. Appl. Microbiol. Biotechnol. 2012.

[34] Wang, J.; Zhao, B.; Yi, Y.; Zhang, W.; Wu, X.; Zhang, L.; Shen, Y. Biochem. Pharmacol. 2012.

[35] Dey, A.; Tergaonkar, V.; Lane, D. P. Nat. Rev. Drug Discovery 2008, 7, 1031.

[36] Dey, A.; Wong, E. T.; Cheok, C. F.; Tergaonkar, V.; Lane, D. P. Cell Death Differ. 2008, 15, 263.

[37] Christian, B. A.; Grever, M. R.; Byrd, J. C.; Lin, T. S. Clin.
Lymphoma Myeloma 2009, 9, S179.

[38] Guo, C.; Gasparian, A. V.; Zhuang, Z.; Bosykh, D. A.; Komar, A. A.; Gudkov, A. V.; Gurova, K. V. Oncogene 2009, 28, 1151.

[39] Sonnemann, J.; Palani, C. D.; Wittig, S.; Becker, S.; Eichhorn, F.; Voigt, A.; Beck, J. F. Eur. J. Cancer 2011, 47, 1432.

[40] Duarte, V. M.; Han, E.; Veena, M. S.; Salvado, A.; Suh, J. D.; Liang, L. J.; Faull, K. F.; Srivatsan, E. S.; Wang, M. B. Mol. Cancer Ther. 2010, 9, 2665.

[41] Wang, J. C. Annu. Rev. Biochem. 1996, 65, 635.

[42] Maxwell, T.; Bates, A. Nucleic Acids Res. 2009, 37, 659.

[43] Topcu, Z. J. Clin. Pharm. Ther. 2001, 26, 405.

[44] Koster, D. A.; Palle, K.; Bot, E. S.; Bjornsti, M. A.; Dekker, N. H. Nature 2007, 448, 213.

[45] Liu, J. K. Chem. Rev. 2006, 106, 2209.

[46] Cai, S.; Sun, S.; Zhou, H.; Kong, X.; Zhu, T.; Li, D.; Gu, Q. J. Nat. Prod. 2011, 74, 1106.

[47] Wei, H.; Inada, H.; Hayashi, A.; Higashimoto, K.; Pruksakorn, P.; Kamada, S.; Arai, M.; Ishida, S.; Kobayashi, M. J. Antibiot. (Tokyo) 2007, 60, 586.

[48] Guo, H.; Hu, H.; Liu, S.; Liu, X.; Zhou, Y.; Che, Y. J. Nat. Prod. 2007, 70, 1519.

[49] Stead, P.; Affleck, K.; Sidebottom, P. J.; Taylor, N. L.; Drake, C. S.; Todd, M.; Jowett, A.; Webb, G. J. Antibiot. (Tokyo) 1999, 52, 89. 